\title{
Innovation in Information Systems applied to the Shoes Retail Business
}

\author{
Vasco F. Teles ${ }^{1}$ and Francisco J. Restivo ${ }^{2}$ \\ ${ }^{1}$ University of Porto, Faculty of Engineering, Portugal, vascoft@hotmail.com \\ ${ }^{2}$ University of Porto, Faculty of Engineering, Portugal, fjr@fe.up.pt
}

\begin{abstract}
Innovation is undoubtedly an essential part of the business process. Innovation management is as important as quality management and customer relationship management for the company success, independently of the business area.

Computer-Aided Innovation is emerging as a strategic domain of research and application to support enterprises throughout the overall innovation process. CAI systems should begin at the creative stage of identifying business opportunities and customer demands, then continue helping in developing phases and, further on, providing help up to the point of turning those inventions into successful innovations in the market.

In retail business, the way the offer is presented to customers is as important as product quality, and it is this combined service that is the main purpose for innovative solutions. Shoe retail business is a significant example where combining creative possibilities with technology-based applications makes it possible to introduce innovation in this branch of activity, particularly in the Portuguese business structure, characterized by the prominence of large shopping malls where the service itself gains an increased meaning.
\end{abstract}

Keywords: Innovation, information systems, shoe retail, creativity, CAI

\section{Introduction}

Retail business has been for long time an ideal field to implement new and innovative solutions, as costumers are very demanding, competition is strong and the business is mature, though constantly evolving.

In this business, innovation has to be very consistent throughout its stages: requirements have to be clear, problem-solving has to be creative, the project has to be very well planned, testing has to be exhaustive and implementation has to be reliable, because normally the store operation isn't interrupted. Since retail has a very large costumer and transaction base, computer-aided innovation acquires 
greater importance in supporting considerably more complex operations, giving the possibility to offer new services to the costumer and implement new concepts in stores, usually in such traditional businesses.

This paper aims to evidence the importance of computer-aided innovation within the retail sector, detailing the above mentioned concepts, when new concepts are installed, new services are offered to the client and to shop assistants and how does all this integrates in companies' information systems. For this we will analyze a project in a retail business, which will implement the concept of costumer self service in shoe stores and providing the shop assistants the necessary tools to an efficient work, all integrated in the company main information systems.

In the next section, there is an insight of the business itself: the reason why it came to light, the market competition, the retail environment in Portugal, its investments in specialized retail, leading to the need to innovate and think ahead, in order to obtain competitive advantage and costumer attention, as supported by Yadav et al. "Detection, development, and deployment require the awareness of external opportunities and the anticipation of future events." [1]

In the following section, the needs and requirements of this business concept are detailed. Requirements are a critical issue in the innovation process and attention must be focused on the output of this activity.

Then it is explained the importance of information systems when innovating to meet the business goals while requirements are checked to compliance of those.

In the final section, the benefits the solution can bring to the business are discussed, and how will they be measured, and the authors discuss what value this analysis can bring to computer-aided innovation, and what value CAI can bring to the retail costumer and the company itself.

\section{The business idea}

In our context, one may define business innovation as an idea or practice that is perceived as being new by the adopting unit [2].

Market competition is fierce and becoming tougher as profit margins get crushed each year. Competitors who have the most optimized processes - business, technological, etc. - throughout the whole value chain are the ones who survive, succeed and grow. This includes dealing with procurement, suppliers, logistics, distribution, and at the end of the chain, the consumer.

Portugal is no exception to this, as the concept of "traditional commerce" is disappearing, while new large shopping centres are built and occupied by stores belonging to large economical groups who have those processes very well driven in order to provide the best products to the costumer at the minimum cost. These investments changed consumer's habits radically in a short period, innovating in many ways: increasing the available choice the client, lowering the average price of goods, introducing convenience of time and location, among others. Invest- 
ments were made to implement large hypermarkets where the costumer is able to find almost everything and specialized retail stores focused on particular sectors.

But why a new shoe store business at this moment? The project we study in this paper is under development to a major retail player in the country, which owns a very large number of stores varying from food to consumer electronics, apparel, clothing, etc. Since several years, shoes have been being sold in company's stores, consistently growing their sales volume, resulting from a well-planned strategy that consists namely of controlling the whole product development process "in house" to self brands as well as selling other suppliers brands. As a result and along with this growth, administration and marketing professionals came to the conclusion that it was possible to open independent shoe stores in order to explore that market, as the whole business process was already integrated in the company.

Although it may seem that the market reached a saturation status, it is always possible to find new ways of doing business, even traditional ones, and achieving good results in a local or global market. In order to do so, innovation is mandatory: thinking ahead to foresee consumers' trends and expectations and implement them before rivals do so is vital to gain advantage, consumer attention and loyalty.

To support this innovation, and specifically in our case-study, information systems technology is used to create instruments which will provide services to the costumers that distinguish these stores from others, as well as tools to help the employees to better assist the clients, and thus providing the company fundamental data to compete, evolve and obtain its profit. Like this one, "some firms and industries are making giant productivity strides through IT and some are achieving new revenue streams and competitive advantages." [3]

\section{Requirements}

Requirements are critical in any project, and even more so in an innovative one as this. Care must be set on business needs, requirements and expectations in order to build accurate technical specifications and provide the correct output.

The business team provided four main requirements for the new stores which we had to follow in order to comply with the main target.

To begin with, the new stores would have be innovative and differentiate themselves from competition, building a new concept of shoe store. This was the key business asset for setting the new stores, so as to find their space in market, capturing costumer and media attention. In such a traditional business, implementing a new chain of stores is a risk namely because of market conditions, so a different kind of store would have to be developed, profiting from the constant search customers do to what is new, as well as those who do not identify themselves with conventional stores, thus trying to gain their loyalty offering good products, fair prices and a new pleasant shopping experience. 
This leads us to another requirement, as in order to provide new and pleasant shopping experience, the customer would have to be autonomous in the store, from the moment he or she enters to the moment the payment is made. As Shaw and Alexander referring to Humphrey and Bowlby, "The development of selfservice techniques (...) represents pivotal retail innovations of the twentieth century that transformed the process of selling and the act of shopping." [4] [5] [6] Meanwhile he or she would have been able to browse the store looking for a product, searching for styles, brands, colours, sizes, locating and trying them. This requirement was set thinking of many factors of today's culture, namely lack of time, independence, eccentricity. With this concept, the costumer wouldn't have to wait in line to ask information to store assistants, saving precious time and energy.

In order to keep store operation costs low, few staff will be available in store, thus the project would have to provide efficient tools to costumer, to the staff and to the company itself. The few staff present in store will be able to perform their tasks in a rapid, efficient and effective way, while assisting clients and operating the store. All these include orders, payments, item location and inventories, among others. These tools will have to be direct, reliable and usable.

The last requirement was that the store would have to be kept in order, as the staff would have to be focused in customer care and operation itself, rather than frequently resetting items in their original places. If a costumer picks up an item and has to carry it more than a very short distance to search information about it, the most probable consequence is that the product will be left somewhere else other than its original location. Consequently, in these new stores, the costumer will have no need of carrying items around and will only need to look up, press a button, stretch an arm or walk few meters, so as to obtain information.

As a baseline of all these, we had in mind what Gammal refers regarding technological innovation as to market success is concerned "Rather than technological failure, the pivotal reason is a lack of true customer relevance - either in the product itself or the way in which it is described and marketed." [7]

\section{Methodology}

\subsection{Creativity process}

As the requirements were set and integrated into the project team, the main question arose, as what kind of devices where going to be necessary to comply with not only business needs and requirements, but also its expectations and constraints. Thus for the moment it was the stage where it was needed to openmindedly come up with ideas. In that sense, a creativity session with people from different backgrounds and competencies in the company was scheduled where it was expected to collect valuable contributions in order to arrive to a solution, as 
supported by King "the consensus assessment of a 'crowd' - a group of people, each having limited knowledge of a subject - can be far superior to expert assessments and even superior to the assessments of each and every individual member of the "crowd.'" [8] The project team naturally had some ideas, but would like to face them with other ones, in order to assess their quality and feasibility.

To this session, it was vital group collaboration, as working together toward a common goal [2] when being apart from current tasks for at least a short period of time, as Yadav et al. sustain when citing Hambrick et al. "that creativity requires some amount of available time and cognitive resources, extremely high levels of job demands may squeeze out novelty and fresh thinking". [1] [9]

The session employed the Synectics process for creative problem solving [10]. This process consists firstly in setting a title for the task, summarizing the problem-solving objective. Starting this title with "How to..." lead to the question "How to implement self service to costumers in shoe stores?".

Afterwards, it is necessary to perform a task analysis alongside with the solution client - the business management, in this case - answering to questions such as: "Why is this a problem or an opportunity?", "What is its brief history?", "What is your power to implement the solution?", "What have you already tried or thought of?", "What is your dream solution?", "What outcome do you want from this meeting?". After this, the problem solving team starts proposing ideas, where exist some basic rules for this section of the work, like suspending judgment in order to open the mind and let others also do it, so every idea is valid; one ought to ponder of what does this problem and the other ideas make one think of, in order to refine ideas and come up with new ones; these should be headlined with "How to..." or "I wish...", as these forms can be particularly useful as they direct resources' attention to where their thoughts would be most valuable. These forms are commonly known as springboards.

A technique called "Excursions" is often used where ideas go round in a closed circle and with that it is possible to obtain fresh ones. It requires the main problem to be set aside and participants to think beyond current constraints. For this, each of the participants is given a specific subject under a theme, like sports or famous people, and tries to associate the subject to the problem, thus generating new ideas. E.g., in a shoe store, Pavarotti would have asked out loud "Where are my Pradas?", and this metaphor originated the idea "Natural language interface to search items in store".

After these idea-creation stages, it is time for the client and the group to select and organize the results. This is completed via a matrix that represents novelty and feasibility in xx and yy axes, respectively, and the ideas are selected based in intrigue and attraction factors, and placed into the matrix according to its less or more novelty and feasibility.

By the end of this process, we have a matrix of possible solutions, as depicted in figure 1, from where the client selects one or several ideas to develop, where only the most relevant solutions are mentioned and the circle size represents an approximate relation between value and complexity. 


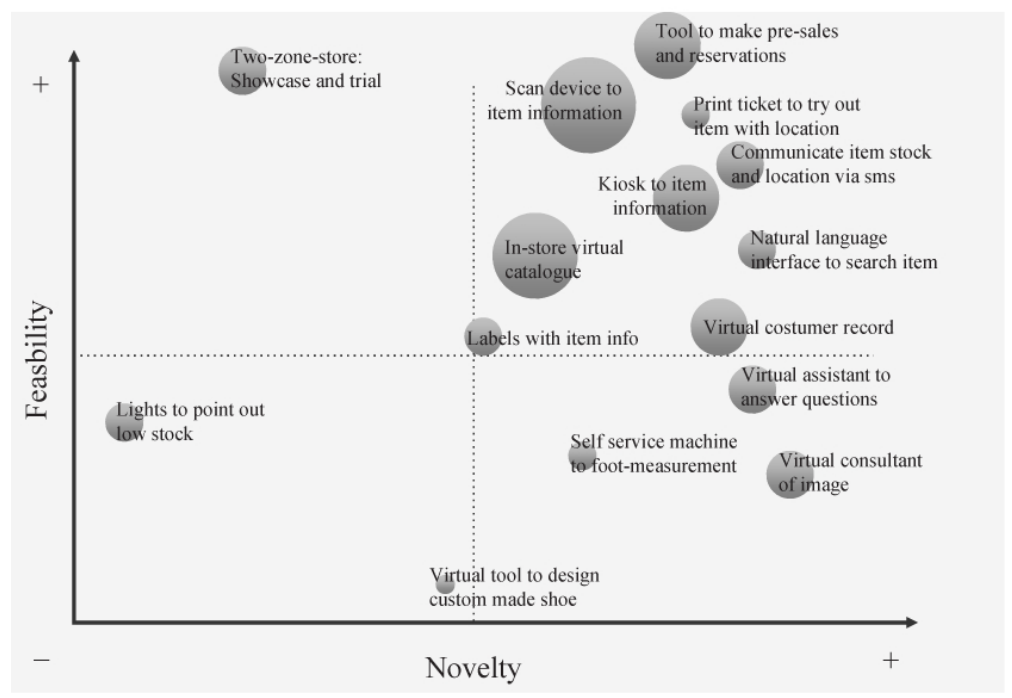

Figure 1 Matrix with relevant ideas resulting from the creativity session

The development is done firstly around the client paraphrases that springboard ideas ("How to..." or "I wish...") decoding any potential metaphors from each idea by asking the author to refine it in other words. After clarified, the idea is constructively evaluated, as the client lists pluses and major concerns about it. This is done once again in the form of "How to...". To overcome those concerns ordered by importance, the client and the group generate ideas, starting with "What you do is...". Finally the client modifies the original idea using the selected modifications, stating it as a possible problem solution. Naturally, this last process can be repeated if necessary, until the client has a comfortable solution.

In the process, creativity only becomes innovation if implemented, so an action macro-plan is set, stating what action is performed by whom and when.

We now briefly analyze some of the more relevant ideas located in the top right quadrant of figure 1, i.e., those which are more novel and more feasible:

- Point of information using barcode scanning devices - this was the selected idea to implement in a short term, complying with business demands. It will use company-based systems in order to provide the expected information and some application developments were needed;

- Interactive kiosk - similar solution to the previous one but with more information, such as virtual catalogue, which would lead to a deeper investment in development, as the whole interface had to be bought/developed as well as the hardware. It is in stand by to a near future;

- Item tags with product information - alike electronic price tags, but with larger displays, thus possibly showing product information as well as stock. It implied a store structure different than the projected, therefore only its concept was worked; 
- Interface in natural language - this was one of the most breakthrough yet feasible ideas. It could be applied in a kiosk and provide the information without the device barrier. It was placed in stand by to some time in future.

Some other final ideas were generated, but these are the most significant and transmit the focus on the problem solving. In the end the idea which will to be implemented led from creativity towards innovation to the business, and fulfilled its balance between needs, requirements, expectations and constraints. These solutions meant to be ambidextrous when balancing between technical and organizational flexibility in response to external conditions and internal needs [1].

To finish with, as supported by Goel and Singh citing Prather et al., this whole process of "creativity and innovation involves the translation of our unique gifts, talents and vision into an external reality that is new and useful. Creativity refers to all activities that involve the generation of ideas. Innovation refers to the implementation of viable business ideas generated as a result of the organization's creativity-supporting culture and structure." [11] [12]

\subsection{Technology}

In this section, we briefly review the business needs, requirements and expectations previously presented, namely the new stores would have be innovative and differentiate themselves from competition, building a new concept of shoe store; the customer would have to be autonomous in the store while few staff would be available; and finally the store would have to be kept in order. So cross-analyzing all these and the solutions resulting from the creativity session, two of them have been considered as feasible in a short to medium term. Some of the others can be applied in the long run, and others still were just good creativity exercises, as often occurs in such processes.

We have seen that, as King supports, "there is also a growing awareness that IT can play an important role in the integration of entrepreneurship and strategy that is necessary if firms are to be quick at identifying opportunities and pursuing them to their advantage" which became clear in this creativity session [3].

Consequently, two of problem solving solutions are: the electronic point of information via barcode scanning and the interactive kiosk. Having this in mind, the solution procurement was started in the market to all its components: hardware, software, kiosks, integrated solutions, and so on. This procurement was made by the team browsing the market and more specifically company's regular suppliers and a major international kiosk fair.

A large range of solutions which could fit in the requirements was found, namely kiosk hardware from a large number of suppliers where software had to be developed and implemented with possibly different types of integration and systems, thus giving vast possibilities to the desired solution, but delivery and development time could be a critical issue; others were integrated and modular kiosk solutions with their embedded applications already focused in a specific business, 
but only one was found dealing with shoe store business, where cost was an issue; some additional type of solutions were barcode scanning devices, some integrated with a screen, where software would have to be developed according to the costumer's systems and requirements, giving the opportunity to apply the company's base solution to price checking and developing it further to comply to the information required to deliver at the store; finally some touch screens devices were analyzed in order to integrate them with an interactive application built in-house to another business, which was possible to adapt and custom develop.

The team them filtered and structured all the information in possible configurations building a proposal which matched the business requirements previously stated, and constraints, such as investments, timings, risk, scalability, equipment and store look and feel. The scenarios were developed around the two main types of solutions: the barcode scanners and the interactive kiosks.

These scenarios evolved along several meetings with the business team and the final decision was taken by the business leader, with technical, design and management inputs. In the end, the chosen solution was the technology already used in company's stores, which employs a bar code scanning device that will display product information, such as name, brand, price and available sizes on its screen, having received that information from company's both checkout and ERP system in real time using web services.

As usually, the decision was taken due to some critical factors described above where this solution was compared to the others presented, and the technology that prevailed was the one which could balance better most of the needs, requirements, expectations and constraints.

\subsection{Competencies}

The search for the right competencies is a key issue in every technology project, and this was no exception. This search was conducted during the final stage of the assessment, where the team already had an approximate idea of what different kind of decisions could face, thus focusing the search. Which competencies from the equipment supplier would be needed in order to install the appropriate software in those equipments? Which competencies would be needed from the software supplier in order to deploy and integrate it in the company's systems? Was the software going to be "off-the-shelf" or custom developed, and if so did the company have internal competencies to build and integrate it?

Alternatively, development could be outsourced, and then again what type of competencies was it needed the development supplier to have in order to create and implement the application the way it was required? Still, with several of these options, internal resources and competencies would have to be employed so as to integrate the solution, whatever it would be, with the company's infrastructure, checkout and ERP systems, among others. This was the common base from which we knew we had to leave in order to build the whole technical team. 


\section{Computer-aided innovation}

Information systems are not a goal by themselves but are useful only when delivering important insight to what they were designed for: decision support, as different information is produced to the costumer, the store assistant and the company in its different profiles - operative, manager, administrator - and handled in diverse manner, by helping the client deciding which item to choose; to support the shop assistant when locating a product; to help operations team decide which items to order; to aid managers and administrators knowing exactly the business status and providing them information to innovate by building new concepts of doing their business, as they are aware they can rely on information systems to go that way.

The project team was very aware of the business goals when projecting possible solutions that resulted from the creativity session, in order to be innovative as required by the business and the store concept itself, but also to be assertive when using information systems as described above. The solution to implement is depicted in figure 2 in a simplified approach.

\subsection{To the client}

Having the previous concepts in mind, the self service system was designed to allow the client to be autonomous when obtaining information about the desired items. Normally, in a shoe store, the costumer has to ask an assistant to find out if the a size is available and other information, sometimes after waiting in a queue, or look around until finding out if there is the correct size or not.

According to the business needs, expectations, constraints and goals, the project will implement in each store several barcode scanning devices with built-in $14 \mathrm{~cm}$ colour screen as these devices are well known to the majority of the costumers, being quite intuitive to operate. The costumer will use this wall-mounted barcode scanner to obtain information, and for that will approach the item barcode to the device laser beam and hears a short beep indicating the product was read and recognized. Some moments after, the information appears on screen.

\subsection{To the store assistant}

Another equally important component of the system as a whole is the one used by store assistants while in their diverse actions during the day. This will include not only customer care in its various types, as providing product information, locating products, suggesting other options to clients, but also current operation as shipment reception, product placement, price tagging, inventory and so on.

While supporting costumers, the assistant will be able to locate exactly where an item is placed, confirm prices, sizes and other product information. To perform this operation, the assistant will have a wireless PDA with barcode scanning capa- 
bility, making it possible to the device to read the item barcode and interpret its EAN code. When interpreted, the information flows the same way as previously referred as for the wall-mounted barcode scanning device was concerned.

This application gives complete mobility to the assistant inside the store to help costumers and to perform routine operational tasks such as receiving product shipments in the background, checking them and confirming the reception, then setting received items inside the store and marking those locations to future reference while assisting costumers. All the information is based in the systems we have previously mentioned, and interface is done via web services once again.

\subsection{To the company}

To end with, store operations, management and company administration rely on various integrated information systems in order to perform their assignments as accurately as possible.

To the operations staff, the ERP is vital so as to analyze stocks, order correct product shipments, minimizing store stock but also out-of-stocks, analyze sales, budgets, margins, legal procedures and others. Alongside, management also rely on information systems, as they have to manage product range, making the right choices to provide the best saleable offer to the costumer and within each product have to negotiate margins, profits and costs, controlling along the way sales in stores so as to perform good results and aim to their sales and profit goals. On top, administration handles data in an aggregate way, therefore usually operates with data warehouse information system which combines data collected. With this system, the company is managed as a whole, even if divided by brands, and steered strategically thus making everyday decisions influencing its course.

The information system will always be a source to decision making, whether it is a costumer in a store checking product information, or the assistant who is locating a product using a portable device connect to the a remote system, or the operations team who supplies the store, its management which decides in which products to bet, or in the end administration who decides the strategy of the company, based in the information provided from all the information systems.

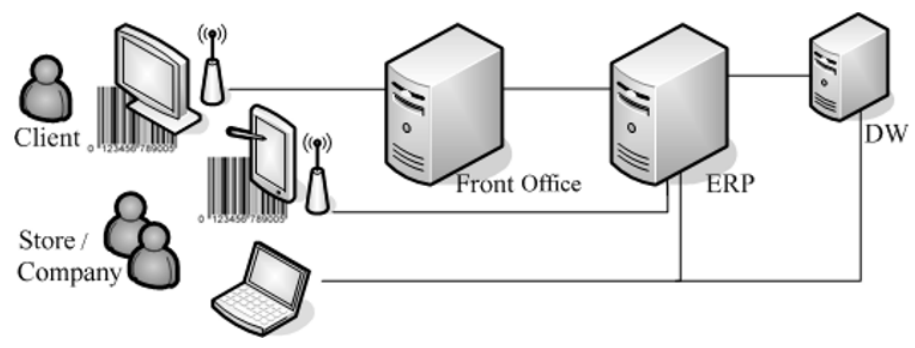

Figure 2 Simplified solution scheme 


\section{Results and conclusions}

\subsection{The goal}

More than a requirement or a business expectation, it is a motivation that the solution is innovative and creates a new concept to shoe shopping in the country. Several analysis have been conducted in order to measure the attractiveness of self service devices in retail, and more specifically amongst this company businesses with quite extraordinary results, as costumers in the country adhere massively to these solutions, as for instance self checkouts in supermarkets.

Of course there is risk where high investment and image values are at stake, as there will be no smooth transition period to these brand new stores. To mitigate this risk, the solution itself and the information systems it integrates with have a very important role providing the client the expected information in a fast, efficient and reliable way. Along with the system, shop assistants also play a part in this equation, as they will need to interact with the client and the system simultaneously, forwarding the first to the latter when possible.

But the solution to be implemented is broader, comprising a powerful and useful tool to store assistants, giving them the possibility to perform their task in a rapid and efficient manner. Following a growing trend, each store will have a small number of employees, thus each one of them will have to do more work in less time. For this productivity issue, information systems are vital so as to provide the required information accurately on time. The based system is widely used in the company, thus having a low risk, but if it fails contingences can be activated.

This solution applies King's concept in which " when IT is focused on producing more flexible manufacturing capabilities, quicker order fulfilment, faster responses to customer inquiries and the like, it usually has direct impact on the bottom line and on 'intermediate outcomes' such as customer satisfaction and quality." [3]

In the end not all the benefits sum up to numbers, like investments, costs, profits, margins, items scanned, items received and so on, and although these are very important and the ones which will rate the project and the solution, there are other intangible gains like company image, costumer fidelity and pioneering that many times "pay" a project.

\subsection{Conclusions}

To conclude with, the authors expect this analysis can contribute to document what is being done when applying computer-aided innovation in the business world, namely in retail sector and shoe stores, to implement new shopping concepts which employ information systems. 
The study case is an example of CAI application in order to satisfy specific business needs and requirements and meet the expectations created around the possible solutions, but also accounting the constraints that naturally emerge. As in all cases, the dream solution would not the one described and implemented but some other less realistic or even impossible for the time being. Thus it is important to point out that computer-aided innovation is built by real developments and implementations and even if it is possible to aim at higher objectives in the future, in many occasions it is preferable to take one step at a time, and implement consistent solutions that can offer some benefits or services to costumer or companies.

These latter are the ones investing large funding to compete in fierce markets and must think ahead of competitors in order to attract consumer attention and loyalty. Therefore, computer-aided innovation is vital to companies if it can provide them tools to compete in the market, bringing added-value to organizations' business. This added-value can be delivered to the company itself, helping it performing its tasks more efficiently and with more information, but also to the costumer, who is the ultimate user to this innovation.

In such a traditional and mature yet rapid and competitive market as retail business, computer-aided innovation makes the difference between those left behind and the ones who profit and grow by offering excellent services to their costumers.

\section{References}

1. Yadav M., Prabhu J, Chandy R.: Managing the future: CEO attention and innovation outcomes. Journal of Marketing, vol. 71, pp. 84-101 (2007).

2. Tarafdar, M, Gordon, S.: Understanding the influence of information system competencies on process innovation: a resource-based view. Journal of Strategic Information Systems, vol. 16 (4), pp. 353-392 (2007).

3. King W.: IT Strategy and innovation: the "IT deniers" versus a portfolio of IT roles. Information Systems Management, vol. 24: pp. 197-199 (2007).

4. Shaw G., Alexander A.: British co-operative societies as retail innovators: interpreting the early stages of the self-service revolution. Business History, vol. 50 (1), pp. 62-78 (2008).

5. Humphrey, K.: Shelf life: supermarkets and the changing cultures of consumption, Cambridge University Press (1998).

6. Bowlby, R.: Carried away; the invention of modern shopping, Faber \& Faber, New York (2001).

7. Gammal, J.: Innovation that pays. Mass High Tech News 30 August (2004).

8. King, W.: IT Strategy and innovation: recent innovations in knowledge management. Information Systems Management vol. 24, pp. 91-93 (2007).

9. Hambrick, D., Finkelstein, S., Mooney, A.: Executive job demands: new insights for explaining strategic decisions and leader behaviours. Academy of Management Review vol. 30 (3): pp. 472-91 (2005).

10. Synectics Breakthrough Creativity. Synectics Limited (2007)

11. Goel, P., Singh, N.: Creativity and innovation in durable product development. Computers \& Industrial Engineering, 35 (1), p. 5-8, Oct (1998).

12. Prather, C., Gundry, L.: Blueprints for innovation. AMA Membership Publication Division (1995). 